\title{
Expanding Touch Input Vocabulary by Using Consecutive Distant Taps
}

\author{
Seongkook Heo, Jiseong Gu, Geehyuk Lee \\ Department of Computer Science, KAIST \\ Daejeon, 305-701, South Korea \\ seongkook@kaist.ac.kr,jiseong.gu@kaist.ac.kr, geehyuk@gmail.com
}

\begin{abstract}
In recent years, touch screens have emerged and matured as the main input interface for mobile and tablet computers calling for extended touch input possibilities. In this paper, we explore the use of consecutive distant taps to expand the touch screen input vocabulary. We analyzed time intervals and distances between consecutive taps during common applications on a tablet and verified that consecutive distant taps can be used conflict-free with existing touch gestures. We designed the two interaction techniques Ta-tap and TaTa-tap that utilize consecutive distant taps. Ta-tap uses two consecutive distant taps to invoke alternative touch operations for multi-touch emulation, whereas Ta-Ta-tap uses a series of consecutive distant taps to define a spatial gesture. We verified the feasibility of both interaction techniques through a series of experiments and a user study. The high recognition rate of Ta-tap and Ta-Ta-tap gestures, the few conflicts with existing gestures, and the positive feedback from the participants assert the potential of consecutive distant taps as a new design space to enrich touch screen interactions.
\end{abstract}

\section{Author Keywords}

Ta-tap; Ta-Ta-tap; consecutive distant taps; command shortcut; touch screen

\section{ACM Classification Keywords}

H.5.2. Information interfaces and presentation (e.g., HCI):

User Interfaces - Input devices and strategies

\section{INTRODUCTION}

Recently, touch screens have emerged as the dominating interface for mobile devices and tablet computers. However, a major downside of touch screen interfaces is their limited input vocabulary. Various touch gestures were designed to handle this problem. For example, touch-andhold is a common gesture for alternative selections. Also,

Permission to make digital or hard copies of all or part of this work for personal or classroom use is granted without fee provided that copies are not made or distributed for profit or commercial advantage and that copies bear this notice and the full citation on the first page. Copyrights for components of this work owned by others than the author(s) must be honored. Abstracting with credit is permitted. To copy otherwise, or republish, to post on servers or to redistribute to lists, requires prior specific permission and/or a fee. Request permissions from Permissions@acm.org.

CHI 2014, April 26 - May 01 2014, Toronto, ON, Canada

Copyright is held by the owner/author(s). Publication rights licensed to ACM.

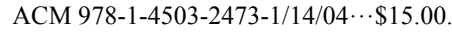

http://dx.doi.org/10.1145/2556288.2557234
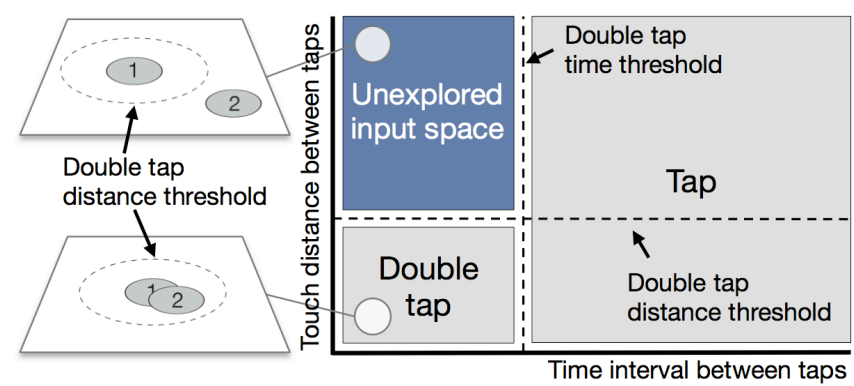

Figure 1. Input space of tap inputs and illustrative examples of double tap and unexplored input space. Gray circles with the numbers 1 and 2 indicate the first and the second taps.

multi-touch gestures such as a two-finger tap or a fourfinger swipe are used for alternative selections or for switching between applications. More recent examples are attempts of using a finger's movement patterns to enable additional touch gestures as well as attempts of enhancing a touch input through additional modalities like the finger contact shape, the touch pressure, and the tapping velocity for a richer touch interaction. Such attempts, however, require additional sensors or signal processing.

We will identify and explore the underutilized gesture space of touch interactions. Although the set of touch gestures is greater than that of mouse inputs, many touch gestures resemble mouse inputs. For instance, tap and click, slide and drag, or double tap and double click are very similar to each other. Among mouse inputs, consecutive clicks, such as a double click and a triple click, are performed only at same locations since it is difficult to move a mouse cursor to a distant location in a short time interval $(500 \mathrm{~ms}$ for the Microsoft Windows default setting [18]). This condition is different for a finger touch. A previous study revealed that a mouse is more accurate when selecting a small target, whereas a finger touch is faster regarding target selection $[7,16,26]$. In addition, when using two fingers, the target selection speed increases compared to the one-finger condition [17], whereas the use of two mice for a symmetric task does not lead to any performance improvement [7].

We anticipate that touch screen users will be able to perform a new set of touch gestures comprised of consecutive taps at distant locations as illustrated in Figure 1. Unlike the double tap gesture, which has only single triggered location information, a set of consecutive distant 
taps adds the information of the second tap's relative location. With the spatial arrangement of the distant taps, the new gesture can be used for triggering alternative touch operations for multi-touch emulation, or for invoking a large gesture set by drawing a dot path with multiple taps.

In this paper, we observe touch operations performed while using applications on a current touch screen device. Furthermore we define a consecutive distant tap and reveal that the consecutive distant tap is an available input space. We then describe two interaction techniques of using consecutive distant taps. We also verify the feasibility of the consecutive distant taps for several applications.

\section{RELATED WORK}

\section{Enriching Touch Input with Touch Behaviors}

The shape of a finger contact has been commonly utilized to augment touch information due to its application without adding any sensors. Such vision-based touch surfaces can detect the whole silhouette of a finger contact utilizing the size of a touch contact $[2,5]$ as well as the orientation of a touch [29, 30]. On a mobile device, the contact area of a thumb touch is utilized [4] to enable richer input for onehanded use.

Finger movement patterns have gained more attention than the shape information, because many mobile devices are equipped with a capacitive touch screen that can estimate the touch area only indirectly via capacitance distribution. For example, MicroRolls [24] has found that the movement of a touch point, while rolling a thumb on a touch surface, is different from that of an existing thumb gesture, such as a drag, swipe, or rub. Bezel swipe [23] uses a drag gesture starting from the bezel of a touch screen to distinguish the initial position of the bezel among similar drag gestures. Bonnet et al. [3] use a similar gesture to that of Benko et al. [2], which is a rocking movement of the thumb. The quick rolling down and up of a thumb is used as an alternative click. Heo and Lee [14] developed a shear force estimation method that utilizes micro-movements of a finger contact while applying shear force. Wagner et al. [28] designed a bimanual interaction technique for touch tablets. Thereby, users can change modes or trigger additional operations with the fingers of the holding hand.

\section{Enriching Touch Input Using Additional Modalities}

Nowadays, mobile devices are usually equipped with more sensors than desktop or laptop computers, such as an accelerometer, gyroscope, magnetometer, microphone, light intensity sensor, and location sensors like GPS. Some studies tried to combine sensor data from built-in sensors on a mobile device to expand the touch input vocabulary. Hinckley and Song [15] proposed the use of motion data obtained by an accelerometer and a gyroscope, and showed various interaction scenarios of using the combinations of motion and touch data. ForceTap [13] uses the built-in accelerometer to estimate the tapping force by calculating the sum of acceleration values caused by the movement of tapping. Serrano et al. [27] presented Bezel-Tap gestures, which consist of a first bezel tap detected by a built-in accelerometer and a following screen tap detected by a capacitive touch screen. Because the first bezel tap does not require touch sensing, Bezel-Tap can be used while a screen is turned off. GripSense [8] uses a built-in vibration motor and gyroscope to detect hand posture and touch pressure by measuring the vibration diminution of the hand. Harrison et al. [11] introduced TapSense, which recognizes finger parts or tools tapping the surface by analyzing the tapping sound.

Force is also a frequently used property. Blackberry storm 2 by Research in Motion [22] has four force sensors installed under the touch screen and measures the normal force to distinguish touch and press. GraspZoom by Miyaki and Rekimoto [19] utilizes normal force for continuous zooming and scrolling. Heo and Lee [12] presented gesture scenarios that use combinations of normal force, shear force, and touch movement. Harrison and Hudson [10] explored possible interaction scenarios to use shear force with touch input.

\section{CONSECUTIVE DISTANT TAPS}

Multiple tap gestures, including double taps and triple taps, are designed like multiple clicks on a mouse. Multiple clicks are usually performed without moving the pointer because moving to a certain location in a short amount of time is difficult, and the user has to rely on visual feedback. On the other hand, we rely on both the visual movement of a real finger, rather than the visual feedback displayed on a screen, and the kinesthetic sense of the finger when using a touch screen. We can switch fingers or use a finger of another hand to input consecutive taps that are not adjacent. However, except for the touch-typing tasks, only multiple taps at the same location are commonly used on a touch screen. A series of consecutive distant taps may open a new input space on a touch screen (Figure 1). In order to determine the possibility of using consecutive distant taps, we first observed the time interval and the distance between taps while using well-known tablet applications.

\section{Time Interval and Distance between Taps}

In order to analyze the taps made while using tablet applications, we had to choose representative applications. Müller et al. [20] studied the use of tablet computers to identify frequent activities or tablet-use contexts. In their result, checking emails, playing games, social networking, looking up information, and watching TV/videos were considered to be frequent uses of touch screen tablets by most participants. Because watching TV or videos does not require frequent interaction, we chose the activities of emailing, gaming, social networking, and looking up information using a web browser. Whereas other studies have mostly considered content consumption rather than content creation tasks, we also added a simple wordprocessing application. A ‘jailbroken' Apple iPad was used in this experiment, and all touch events and the use of the 
touch screen keyboard while using the selected applications were logged. Twelve students with an average age of 21.1 years ( 6 female and 6 male) participated in the pilot study. All participants were experienced with touch screen devices, and eight of them were currently using a tablet. All participants reported that they did not experience any difficulties using the applications. Participants were compensated with approximately $\$ 7$ for their time.

In the pilot test, participants were asked to use the selected applications freely for 5-10 minutes. For the emailing and content creation tasks, we asked participants to perform predefined tasks rather than to use them freely. While using the Mail app, which is a built-in email client on an iPad, we asked participants to browse and search through emails and draft a short reply to a specific email. For the web-browsing task, the participants were asked to freely browse and search web pages with the Safari app. The Facebook app was used as an example of a social networking scenario. Fruit Ninja and Juke Beat were used for the gaming scenarios. Both gaming applications require frequent touches; Fruit Ninja required frequent short slides, and Juke Beat required frequent taps for each beat in a song. For the content creation task, the participants were asked to make a copy of a sample document, which included a picture, normal, bold, and different-sized fonts, and aligned texts, using Apple Pages.

We collected 14,193 touch events in total. These touch events were grouped into four categories: tap, typing tap, slide, and multi-touch operations. Each category had 4400, 4479,4908 , and 406 touch operations. We analyzed the time interval and the distance between consecutive taps. Figure 2 shows the distributions of the time interval and the distance of non-typing taps and typing taps. The time interval axis was cropped to $3 \mathrm{~s}$ for better visualization of the short-interval taps, which were of interest to us. We found that the non-typing taps with a short time interval $(<0.5 \mathrm{~s})$ were adjacent to each other $(<9.6 \mathrm{~mm})$ and that there was no distant tap with a short time interval. This
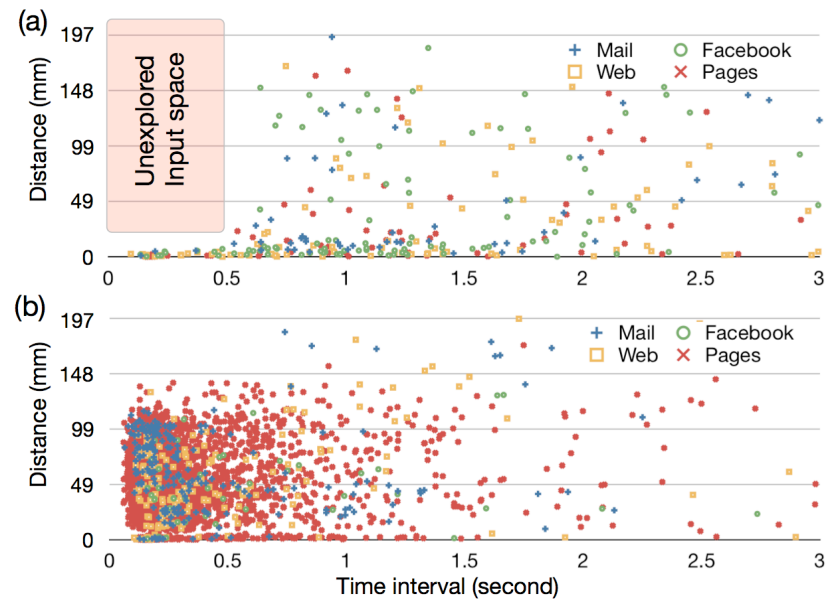

Figure 2. Time interval and distance distributions of (a) taps and (b) typing taps resulted in an unused input space becoming annotated as shown in Figure 2a, and we called the taps in this area "consecutive distant taps." In contrast, $87.2 \%$ of the typing taps had a time interval of less than $0.5 \mathrm{~s}$ (Figure 2b). This revealed that the users were capable of making consecutive distant taps. Taps made from the Juke Beat application were spread throughout the entire area, including the shortinterval distance area. However, the gaming applications were special cases that were difficult to generalize and touch operations frequently used in the games conflicted with double taps or multi-touch gestures. Thus, we omitted Juke Beat taps from the graph in order to emphasize the unexplored area. We also excluded Fruit Ninja taps because only a few number of taps, which were used for menu selections, occurred per person.

\section{Using Consecutive Distant Taps}

We verified that the consecutive distant taps belonged to an unused input space. The next step was designing interaction techniques with those consecutive distant taps. While there could be a continuum of application possibilities, we explored the two extreme ends. On the one extreme, we used two consecutive distant taps that we called Ta-tap. A Ta-tap can be used for defining two control points for a zooming or rotating operation. It may also be used for instantiating a GUI control that can be defined with two points such as a scroll wheel control or a pie menu. On the other extreme, we used an arbitrary number of consecutive distant taps to form a spatial gesture. For instance, we can make a constellation gesture based on key locations on a QWERTY layout. Numerous spatial tap gestures could be defined and used for invoking a command. We called such a spatial tap gesture Ta-Ta-tap. In the following two sections, we explore the possibility of the two extreme cases of Ta-tap and Ta-Ta-tap, respectively.

\section{TA-TAP: USING CONSECUTIVE DISTANT TAPS FOR ONE-HANDED TOUCH SCREEN USE}

We define a Ta-tap operation as a set of two consecutive distant taps. Ta-tap can be useful for a one-handed touch screen use because its performance does not require multiple fingers. As shown in Figure 3, Ta-tap consists of two stages: activation and manipulation. When two consecutive distant taps are detected, a GUI control that consists of two handles (circles) at the two taps is displayed on the screen. The users can then drag one of the handles to use the control. Because the manipulation is performed with a drag gesture, we modified Ta-tap to be activated with the touchdown of the second tap. A novice user can perform two taps to check the manipulation handle, and then perform the manipulation. With this modification, expert users can seamlessly perform manipulations with a drag gesture.

\section{Ta-tap Use Scenarios}

We developed three use scenarios of using Ta-tap, which are multi-touch emulation, virtual scroll wheel, and pie- 
menu invocation. In the first scenario, two consecutive distant taps are regarded as two touches. When a user performs Ta-tap, a handle with two circles at two tap locations appears on a screen as shown in Figure $4 \mathrm{a}$. The user can move one of the circles to zoom or rotate an image or a map with an anchor point at another circle location. If a user performs drag instead of the second tap, the first tap location becomes an anchor and the drag location scales or rotates the picture. With this technique, users can perform multi-touch operations with a single thumb.

In the second scenario, users can make a virtual scroll wheel on a screen with a Ta-tap. As shown in Figure 4b, the first tap location defines the center of a scroll wheel, and the second tap location determines the radius of the wheel. Users then circularly move their finger on a wheel to continuously scroll a long content, without repeatedly flicking their finger. It is also possible for a user to preform a drag instead of a second tap, to access the scrolling function, instantly.

Ta-tap can also be used for invoking a pie-menu. Accessing the menu at the top or bottom of the screen can be hard for single-handed use and it becomes harder on larger touch screen devices. In contrast, invoking a pie-menu with $\mathrm{Ta}$ tap allows users to run commands at the 'sweet spot' of the touch screen, the center area. As shown in Figure 4c, the first tap location sets the pie-menu location, and the second touch moves the handle to select a menu item. Users can cancel the pie-menu by locating the second touch handle to the pie-menu center.

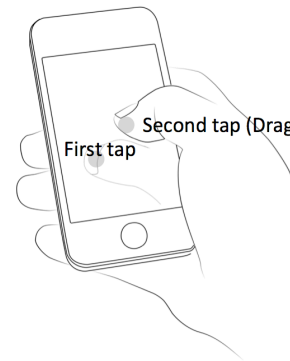

(a)

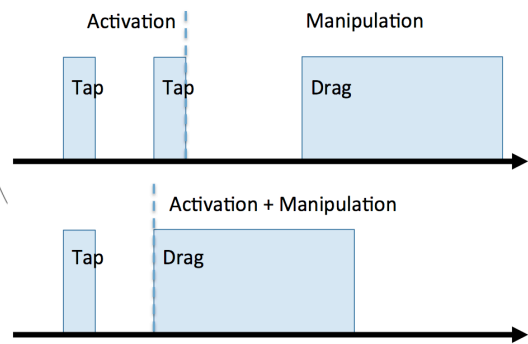

(b)
Figure 3. (a) Performing Ta-tap, (b) integration of Ta-tap and drag operation

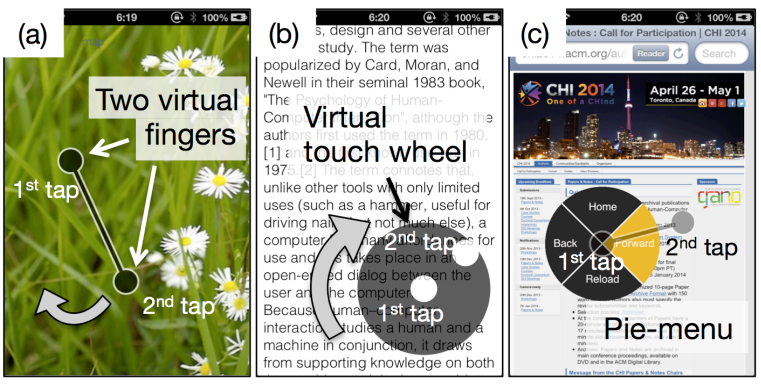

Figure 4. Using Ta-tap for (a) multi-touch zoom / rotate, (b) virtual scroll wheel, and (c) pie-menu invocation

\section{Feasibility Test}

We conducted an experiment to evaluate the feasibility of Ta-tap. The experiment was based on two questions:

- Can users perform consecutive distant taps within 500 ms?

- Can users distinguish between a double tap and a Ta-tap?

The first question is to check whether the $500 \mathrm{~ms}$ threshold, which is a time threshold for double tap detection for many systems, is enough for Ta-tap with a single thumb so that we can use Ta-tap without increasing the existing double tap detection delay. Even though a user can perform Ta-tap, it can be problematic if performing distant taps are too difficult to perform so that the taps are not far enough from each other to be discriminated from double tap. Thus, we checked whether Ta-tap is a gesture that can be differently performed by a user.

\section{Task and Procedure}

The experiment consisted of two tasks. The first and second tasks were designed to answer the first and second questions, respectively. Figure 5 shows the experimental setting and experimental program user interface. The first task was a consecutive tapping. When the experiment started, two circles were displayed at random locations as shown in Figure 5b. Two targets were located at more than $7.7 \mathrm{~mm}$ (50 pixels on iPhone) from each other, and number 1 and 2 were written to indicate the tapping sequence. The task consisted of five blocks and each block had 20 trials. The second task was a gesture-performing task. Before starting this task, we briefly introduced how to use two different gestures: double tap and Ta-tap. Participants could try two gestures for a while ( $<3 \mathrm{~min})$. The target gesture was displayed as an icon, double tap as two concentric circles and Ta-tap as two distant circles as shown in Figure 5c. Participants were asked to perform the displayed gesture anywhere on the screen. The recognized gesture was shown on the screen after the participant performed the gesture. Participants were asked to perform 20 trials in 5 blocks.

We recruited seven participants ( 6 male and 1 female) with an average age of 26.14 years. All participants were righthanded and were familiar with touch screen mobile devices. The participants received $\$ 5$ each for their participation.

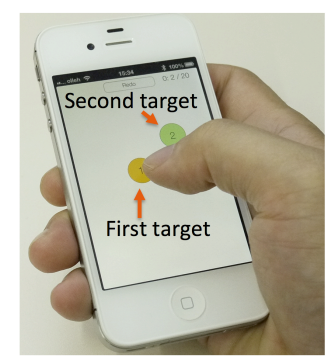

(a)

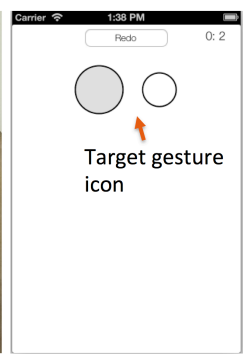

(b)

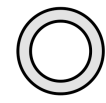

Double tap icon

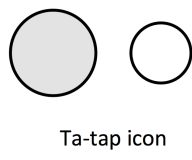

(c)
Figure 5. (a) Experiment settings and screen configurations of experiment 1 and (b) experiment 2. (c) Target gesture icons 
The experimental program was implemented in Objective $\mathrm{C}$, and runs on Apple iPhone $4 \mathrm{~S}$ with a 3.5-inch display. All the participants completed the experiments while being seated, using the device with a thumb of a single hand.

\section{Result}

The results revealed that the interval between the two taps performed by all the participants was less than $500 \mathrm{~ms}$, with an average of $231.1 \mathrm{~ms}$ and the maximum of $432 \mathrm{~ms}$ (see Figure 6). In the second experiment, the participants correctly performed $99.6 \%$ (3 errors $/ 700$ trials) of the double taps and Ta-taps. The three errors were attributed to the participants' mistakenly performing different gestures instead of the target gestures. As shown in Figure 6, the double taps and Ta-taps could be clearly distinguished by the distance threshold. The participants used three applications and were asked to comment on the usability of the new gesture. All participants answered that the new gesture was easy to learn and use. Multi-touch operations and the virtual scroll wheel scenarios were preferred among the three use scenarios. The participants commented that the multi-touch operation was natural and easy to understand and that it would be useful for one-handed use. For the virtual scroll wheel, the participants liked that they did not need to repeat the scroll with their thumb when scrolling a long page.
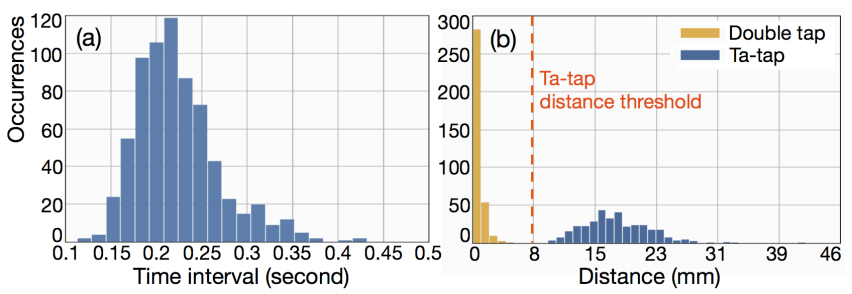

Figure 6. Histograms of (a) time intervals between two taps from the first task and (b) distances between two taps from the second task

\section{TA-TA-TAP: USING CONSECUTIVE DISTANT TAPS AS COMMAND GESTURE}

Another possible use of consecutive distant taps is to define a spatial gesture. We can define a constellation gesture with a series of consecutive distant taps. Numerous spatial tap gestures, that we call Ta-Ta-tap, can be defined this way and be used for invoking a command. The first two taps in Ta-Ta-tap should be at a distance to avoid conflict with double tap. While Ta-Ta-tap can open a huge input space, it poses a scalability problem for gesture designers and a learnability problem for users. It is not easy to come up with a distinct $T a$-Ta-tap gesture for a new command. It is also difficult for users to remember numerous Ta-Ta-tap gestures.

As a means to handle the scalability and learnability problems, we decided to exploit the spatial knowledge that we are familiar with: QWERTY typing. Different from stroke gestures, words themselves have meanings, making it easier to learn them and to form a large set. Gustafson et al. [9] already showed that spatial knowledge is transferred from physical devices to an imaginary interface. Since QWERTY typing without visual cues was also investigated by Findlater et al. [6], we assume that the spatial information of the QWERTY layout and the sequence of typing could also be transferred to an invisible flat surface.

In order to design an algorithm of using consecutive distant taps on the QWERTY layout, it is necessary to observe the patterns of blind QWERTY typing on a tablet.

\section{Investigation on Blind QWERTY Typing}

In order to use the sequences of consecutive distant taps, the time threshold to change the operation mode to the consecutive distant tap detection mode should be defined. We conducted an experiment to measure the time interval between the first and the second tap while typing short commands to determine the time threshold. We chose 15 commands, namely save, load, left, right, center, font, color, bold, italic, zoom, copy, cut, paste, find, and replace. These commands were displayed on a screen in a random order, and all commands appeared five times each.

This experiment was performed with the previous pilot study on time interval and distance in the consecutive distant taps section, thus the same participants were recruited in this study. This study had a $2 \times 2$ betweensubject design with two factors: key layout visibility and number of typing fingers. Half of the participants typed commands with a keyboard layout on a screen and the other half typed without keyboard layout. Six participants typed commands with two thumbs while holding the device. The remaining six typed with all fingers while placing the device on a table. The participants were not informed about the purpose of the experiment. Participants performed all tasks while seated and resting their arms on a table. Further, the Apple iPad 3 was used for the experiment.

Participants of the without-keyboard-overlay condition assumed that there is an on-screen keyboard. There was a keyboard-shaped overlay for the participants who performed the test under the with-keyboard-overlay condition. These participants were also told that the keyboard was not real, so they did not need to be accurate. The position and the timestamp of the taps were logged.

The time interval between the first and the second tap was important because the first two touches decided the mode changes. Figure 7 shows the histogram of the time interval between the first and the second taps. The average time interval was $234.9 \mathrm{~ms}$ when there was a keyboard overlay and $230.7 \mathrm{~ms}$ when there was no overlay. The value at $95 \%$ percentile was $399.9 \mathrm{~ms}$ for the keyboard-overlay condition and $333.2 \mathrm{~ms}$ for the no-overlay condition. Even though the keyboard overlay condition had a longer tail as we can see in Figure 7, most of the consecutive taps were closer than $400 \mathrm{~ms}$. Thus, we set the threshold to $500 \mathrm{~ms}$, which is a threshold of the double tap detection and is sufficiently long to detect typing-like taps. 


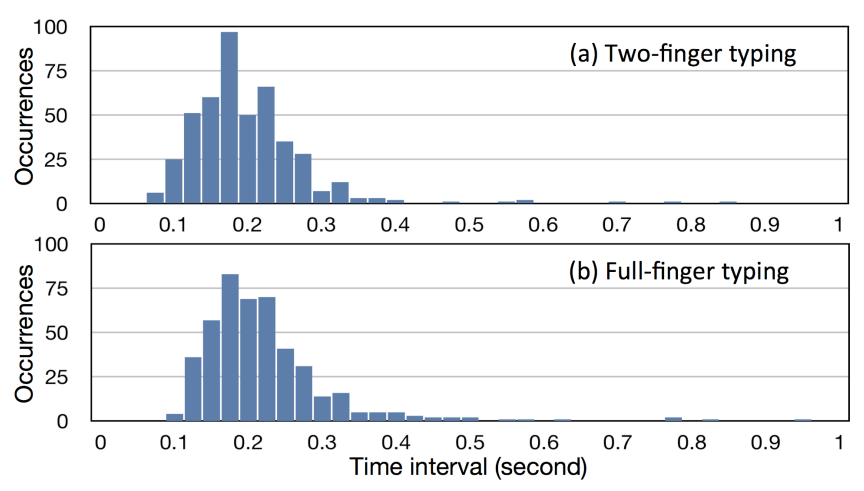

Figure 7. Time interval between first and second taps of (a) two-finger typing (b) full-finger typing

\section{Gesture Recognizer}

There has been intensive research on gesture recognition techniques. Gesture recognizer by Rubine [25] recognizes gestures based on statistical features and needs a large number of sample gestures for training. DTW [21] can recognize gestures independent of the time variance and exhibits high accuracy with a small set of templates; however, it requires a relatively long computation time. \$1 gesture recognizer [31] provides a simple algorithm and exhibits high accuracy with a small number of templates. However, it still requires rotation and scaling to compensate for the individual differences.

Compared to gestures used in the aforementioned research, gestures made with consecutive distant taps have more constraints. When using a touch tablet, people assume the width of the screen to be the width reference of a screen keyboard, making the width fixed. Further, the tablet itself becomes the rotation reference as the conceptual model of an imaginary keyboard is horizontally aligned. Because the QWERTY layout is familiar to many people, their spatial knowledge of the keyboard is transferred to the imaginary keyboard. Since each vertex is made by a single tap, the resampling of in-between samples is not required. Thus, we can build a simpler gesture recognizer, which removes the resampling, translation, scaling, and rotation requirements from that developed by Wobbrock et al. [31]. Further, we can calculate the average distance $d_{i}$ between the points of a consecutive distant tap sequence $\mathrm{H}$ and the $i^{\text {th }}$ point of templates $\mathrm{T}_{\mathrm{i}}$ as follows:

$$
d_{i}=\frac{\sum_{k=1}^{N} \sqrt{\left(H[k]_{x}-T[k]_{x}\right)^{2}+\left(H[k]_{y}-T[k]_{y}\right)^{2}}}{N}
$$

Gesture templates are essential for gesture recognizer systems, and the generation of templates is time consuming. Gesture templates for Ta-Ta-tap can be automatically generated on the basis of the position of each key on the QWERTY layout. When a word or a set of words is given, the first character becomes the origin and the locations of the following characters can be calculated with respect to the origin.
We implemented the proposed algorithm using Objective C. When a tap was detected, the system saved the current touch location and waited for the next tap for $500 \mathrm{~ms}$. If the next tap was detected within $500 \mathrm{~ms}$ and the distance between the taps was larger than 50 pixels $(9.6 \mathrm{~mm}$ on iPad and $7.7 \mathrm{~mm}$ on iPhone), the system entered the Ta-Ta-tap mode. In the Ta-Ta-tap mode, every relative tap location to the first touch location was added to the consecutive distant tap sequence.

We did not consider preventing false positives. In the real use, the algorithm can display command candidates to the user, and the user may select among the candidates or dismiss false positives (see Figure 12).

\section{Experiment 1-Performance Evaluation}

In this experiment, we evaluated the recognition performance and the computation time of the Ta-Ta-tap algorithm. We performed the experiment twice, once on the iPad and another time on the iPhone.

We recruited participants who can type QWERTY-layout keyboard blindly. Ten people ( 3 female, 7 male) with an average age of 22.8 years and eight people ( 5 female, 3 male) with an average age of 20.75 years were recruited in this experiment for iPad and iPhone, respectively. The participants were compensated approximately $\$ 5$ for their effort. All participants were experienced with touch-typing on a mobile phone, and six participants for the iPad experiment were familiar with typing text on tablets. We also asked the preferred posture they used for typing text. All participants for the iPhone experiment preferred twothumbs typing with their hand and eight over ten participants for the iPad experiment preferred ten-finger typing.

The experimental application was developed in Objective C and run on an Apple iPad 3 and an iPhone 4S. All participants performed the experiment while being seated and resting their arms on a table. Figure 8 shows the poses of the task execution. In the iPad experiment, half of the participants completed the tasks with two thumbs, and the other half typed with ten fingers. For the iPhone, all participants performed tasks only with two thumbs.

\section{Task and Procedure}

We collected 200 app names from the Apple App Store's Top Charts, 100 names of free apps and 100 of the paid apps. We then removed special characters and numbers from the app names because our algorithm only supports alphabetic characters and spaces. We also excluded app names that had many numerical characters. As a result, we had a total of 192 app names.

The experimental task was designed to be similar to that of Findlater et al.'s study [6]. The participants started the experiment right after a short instruction, without any practice. As shown in Figure 8a, a randomly chosen app name is displayed on the screen. When a participant tapped 
the screen, an asterisk feedback was shown to indicate the number of taps they entered. The participants proceeded to the next trial by tapping the button with the 'Next' label that was activated as soon as they completed entering the name without mistakes. When they had made a mistake, they could retype the word by tapping the 'Again' button. In order to prevent users from tapping the 'Next' button instead of 'Again' button accidentally, we enabled 'Next' button only when the number of taps matched the number of characters of the task word. The purpose of the experiment and the recognition results were hidden to the participants. Each participant performed 100 trials.

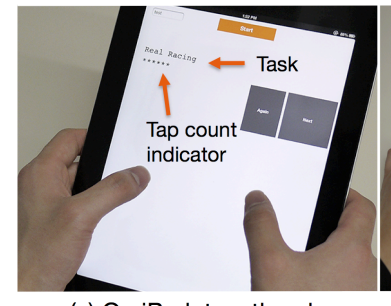

(a) On iPad, two thumbs

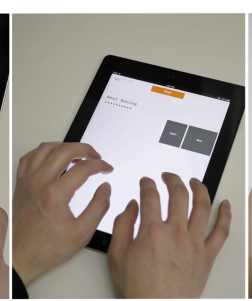

(b) On iPad, all fingers

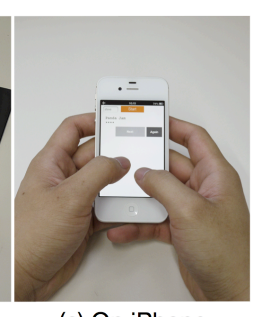

(c) On iPhone, two thumbs
Figure 8. Tapping poses in the experiment

\section{Result: Gesture Recognition Performance}

The algorithm calculated the average of distances for every trial and every consecutive tap that exceeded a single tap. The recognition result is summarized in Table 1 . In the case of the tablet use condition, 493 sets $(98.6 \%)$ and 490 sets (98.0\%) of Ta-Ta-taps over 500 trials matched to the gestures with a minimum average distance for two-thumbs and for all fingers conditions, respectively. Including gesture candidates with second-minimum average distances, the recognition rates for the two-thumb condition and full-finger condition increased to $99.6 \%$ and $98.4 \%$, respectively. Recognition performance was similar with the iPhone use condition. Among 800 trials, the best-matched templates of 787 trials $(98.4 \%)$ matched to the task word. The recognition ratio increased to $99.1 \%$ and $99.4 \%$ with candidates with including second-minimum average distances and candidates with including both secondminimum and third-minimum distances, respectively. The average computation time for matching one gesture was $33.6 \mathrm{~ms}$ on the iPad and $29.4 \mathrm{~ms}$ on the iPhone. This means that the algorithm could match 192 gestures 30 times per second, and it had a possibility of real-time processing.

Typing all letters may not be feasible in a real use scenario, because the names of some applications are longer than 20 characters. Hence, recommending commands as tapping is necessary. We measured the minimum required number of taps to be correctly classified. Figure 9 shows the histogram of the number of characters including spaces of app names used in the experiment, and the number of taps required to achieve the correct recognition. As a result, $82.3 \%$ of $\mathrm{Ta}$ Ta-taps were correctly classified within four taps.

As shown in Figure 10, users could make a similar pattern for the same term without any keyboard layout visualization. Even though the absolute locations are different, the recognizer still could recognize patterns correctly.

\begin{tabular}{|l|c|c|c|}
\hline Number of Candidates & $\mathbf{1}$ & $\mathbf{2}$ & $\mathbf{3}$ \\
\hline \hline Tablet, Two thumbs & $98.6 \%$ & $99.6 \%$ & $99.6 \%$ \\
\hline Tablet, All fingers & $98.0 \%$ & $98.4 \%$ & $98.8 \%$ \\
\hline Phone, Two thumbs & $98.4 \%$ & $99.1 \%$ & $99.4 \%$ \\
\hline
\end{tabular}

Table 1. Recognition rate by the number of gesture candidates

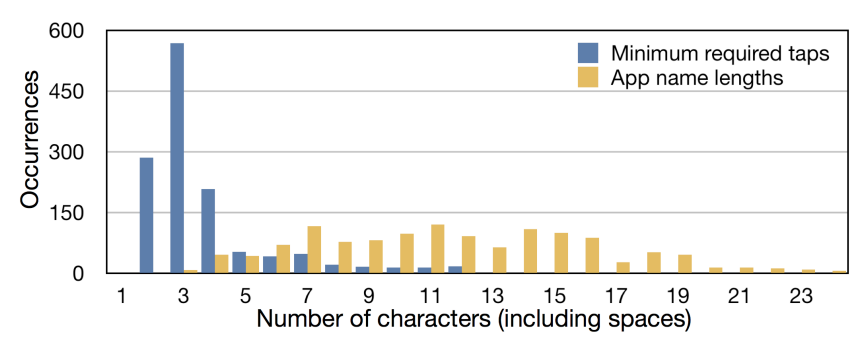

Figure 9. Histogram of app name lengths and minimum number of taps for correct recognition

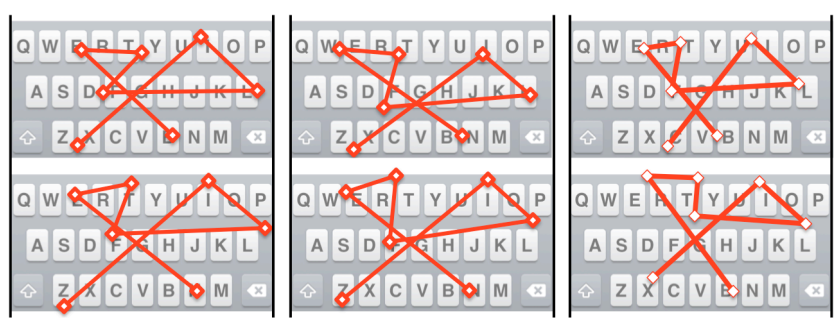

Figure 10. Gesture patterns of "Netflix" of four users from the iPhone experiment, which are all correctly recognized. We overlaid iPhone screen keyboard for better understanding.

\section{Experiment 2-Gesture Conflict}

Although we observed that the input space of consecutive taps is not being used on many applications, it is necessary to check whether they create a conflict with the other touch gestures. We conducted an experiment to check the conflict of the Ta-Ta-tap gesture with touch gestures, which are tap, double tap, and drag. Eight individuals (1 female) with an average age of 24.75 years participated in this experiment. The Apple iPad $3^{\text {rd }}$ generation was used, and the experimental application was implemented in Objective C. All the participants completed the tasks while remaining seated; however, we did not ask the participants to assume a specific posture or to use a specific finger.

\section{Task and Procedure}

In this experiment, the participants were asked to perform touch gestures following a short instruction displayed on the screen. The tasks included tapping and double tapping a box, dragging an item, and typing a command using Ta-Tatap. The tasks were displayed in a random order. The experiment consisted of three blocks, and each block had 20 trials ( 5 trials for each gesture). The screen had 20 target 
boxes aligned in 5 rows and 4 columns, and all the target boxes responded to touch gestures. Hence, we could log all the gesture events.

\section{Result}

Among the 480 trials, a tap, three double taps $(2.5 \%$ of 120 double taps), two Ta-Ta-taps (1.67\%), and one drag $(0.83 \%)$ were misclassified as other gestures. One tap and two Ta-Ta-taps were classified as drag gestures, which was attributed to the sliding of the finger when it touched the screen surface. Three double taps and a drag were classified as taps. No touch gesture was classified as Ta-Ta-tap, and the misclassification of Ta-Ta-tap was not higher than that of the other gestures.

\section{Discussion}

Ta-Ta-tap relies on the user's recall capability. Different from the recognition-based user interface, recall may cause difficulties remembering exact commands. For example, if a user who may want to add a meeting to her calendar should have typed 'Schedule' instead of entering the word 'Book'. Because the Ta-Ta-tap algorithm can make a gesture template based on the characters in a word, we can generate multiple gestures with synonyms for a single function.

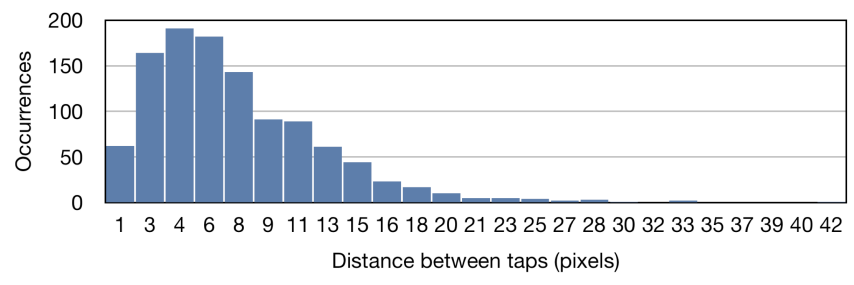

Figure 11. Histogram of double tap distances

Using Ta-Ta-tap on a small-sized touch screen can cause conflicts with double tap since the distances between adjacent keys are shorter (approx. $4.9 \mathrm{~mm}$ on the iPhone) than the double tap distance threshold (approx. $6.9 \mathrm{~mm}$ on iPhone, experimentally determined). We thought that the double tap distance could become shorter than the current setting. We had a brief study to determine the distance between two taps while performing double taps. 14 participants performed 100 double taps on randomly displayed targets. Figure 11 shows the histogram of distances between two taps performed by the participants. The result showed that more than $99 \%$ of the double taps have a distance between the taps that measures less than 3.9 $\mathrm{mm}$. Thus, we can lower the double tap distance threshold for Ta-Ta-tap enabled controls on small-sized touch screen devices.

\section{FOCUS GROUP}

We conducted a focus group to receive usability feedbacks, to reveal usability issues, and to look for possible application scenarios. We recruited five undergraduate students (four male and one female), since the recommended number ranges from three to seven [1]. All participants were experienced users of touch screen mobile devices, and the four of the participants were familiar to touch tablets. At the beginning of the focus group interview, we gave a brief instruction on the consecutive distant tap input and demonstrated Ta-tap and Ta-Ta-tap. Then, participants were asked to test Ta-tap and Ta-Ta-tap with the selected applications of an image viewer with $\mathrm{Ta}$ tap multi-touch emulation, a text reader with Ta-tap scroll wheel, and a web browser with Ta-tap pie-menu which are aforementioned in the Ta-tap section. We also developed a mock-up web browser with Ta-Ta-tap functionalities. Figure 12 shows the application. Users can type a command on a web page view. When two consecutive distant taps are detected, the system blocks the touch event delivery to web view control and enters the Ta-Ta-tap mode. In the Ta-Tatap mode, a dialog window with four buttons appears approximately $2 \mathrm{~cm}$ above the highest touch location. At every tap, the titles of three buttons from the left side on a dialog window changes to top three gesture candidates. Thus users do not have to type all letters but select when the intended command appears. The last button is to cancel the Ta-Ta-tap mode. Then, users may select a function by choosing a button. In our implementation, we applied a multi-stage command system. Users may type "scrap" and confirm to scrap the page. Users can type "run" to confirm, then type "youtube" and confirm to run the YouTube application. To make a reminder, they can type "new" and confirm, type "reminder" and confirm, then write down a reminder on a pop-up window. Participants used the Ta-tap applications on an iPhone and the Ta-Ta-tap application on an iPad.

After using the applications, we asked the participants to comment on the following questions: could you use the new interaction technique; did you have any difficulties using it; what were pros and cons; and are there other scenarios that this technique might be useful for. All participants answered that they could easily find how to use it and they felt that both Ta-tap and Ta-Ta-tap were intuitive to use. One participant answered that he may have had difficulties learning the techniques if there was only a text manual, and he commented that a short video manual would be better to understand. All participants also told that both techniques are useful. The preferred use scenarios using Ta-tap was different for each participant, and no scenario was significantly preferred. One participant commented that the use scenarios of Ta-tap were useful, but only one scenario could be performed at a time. For example, when using a web browser, users may want to zoom in a web page, scroll the page with a wheel, and perform shortcut menus with a pie menu. She suggested combining double tap and consecutive taps, which we can call as consecutive distant double taps, to add to the Ta-tap mode. Four participants liked and had no difficulties of using Ta-Ta-tap, but one participant reported that he failed to memorize the QWERTY layout so that he had difficulties using it. 
The participants gave us many ideas for using new gestures. One participant suggested that the tapping locations might form a rectangle so that he can capture a screenshot of a selected area. Another participant told that the second tap of Ta-tap is usually manipulated with a drag and confirmed by a finger lift after the drag, making the location of the second tap less important. So she suggested using the relative location of the second tap to enable multiple mode Ta-tap operations. Other participants commented that the Ta-tap would be also useful for rate-controlled scrolling, with the relative position of the second tap to the first tap.

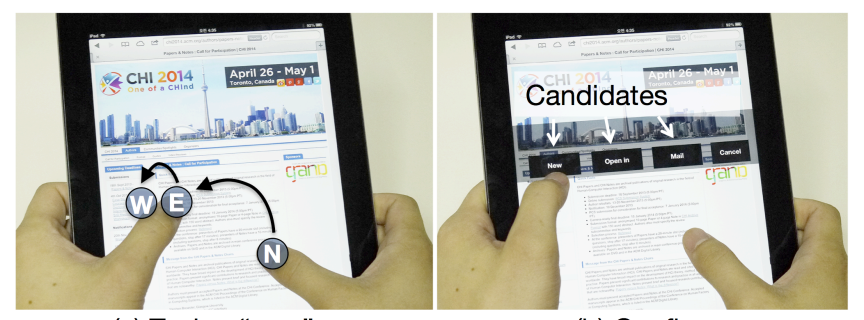

(a) Typing "new"

(b) Confirm

Figure 12. Mockup web browser application with a Ta-Ta-tap interaction technique

\section{DISCUSSION}

To open up the unused input space of consecutive distant taps, working within $500 \mathrm{~ms}$ delay threshold is unavoidable. In fact, current tablet systems, such as an iPad, already have $500 \mathrm{~ms}$ of delay to detect double taps and the delay exists on double tap enabled controls. Apple tried to reduce the feeling of the delay by giving a visual feedback before triggering an action. For example, the Safari app uses a double tap gesture to zoom a paragraph on a web page. It has a delay to detect the double tap and triggers the tap gestures $500 \mathrm{~ms}$ after the gesture. The system shows a visual feedback indicating that a tap is performed when the first tap was detected so that the user can notice that his input has not been ignored. It may help users to experience a shortened delay. We use a similar approach. When a user taps a screen, a visual feedback shows a preview of a single tap operation.

As we observed in the pilot test, some game applications use consecutive taps at various locations. Thus, there can be conflicts between gameplay taps and consecutive distant tap gestures. It is an unavoidable limitation, while gameplay uses maximum input capabilities. The problem already exists with currently used gestures. Tap-and-hold and double tap gestures are also being used in many game applications. However, despite the limitations, we still perform well for most of the applications. With an option to enable or disable the consecutive tap gesture, the gesture potentially will be useful for many applications.

In the focus group interview, we learned that participants considered multiple mode Ta-tap operations to be better, to which we agreed. There can be three ways to add modes to the Ta-tap operations. First, we can use the relative position of two taps. As one participant commented in the focus group, the relative location of the second tap was not utilized. Previous work [32] also showed that utilizing the relative position of the pen stroke to the initial tap or stroke can increase the input vocabulary of marking menus. Thus, we may change modes through the relative direction of the second tap location or through the distance between two taps. Secondly, we can utilize the time to change a mode. Since we are already familiar with utilizing the time on a touch screen in a touch-and-hold gesture, adding the time factor to Ta-tap will be also feasible. Finally, we can use more taps. We introduced Ta-tap as the simplest way of using consecutive distant taps, but there can be slight modifications. In the Ta-tap use scenario, we emulated twofinger gestures with two consecutive distant taps. In a similar manner, three or four taps can emulate three- or four-finger gestures like the ones used on the iPad to switch applications. Two distant taps can be two distant double taps.

\section{CONCLUSION}

In this paper, we revealed an unused input space of consecutive distant tap gestures, and showed through a user study that the input space has not been used. We developed two interaction techniques utilizing consecutive distant taps, and verified their feasibility through a series of experiments. Participants reported that they could use consecutive distant taps with ease. The experiment also showed that the simple gesture recognizer for QWERTYlike consecutive distant tap gestures had a recognition rate of higher than $98 \%$ within approximately $30 \mathrm{~ms}$ of the average computation time. More than $82 \%$ of the 192 application names were recognized within four taps. The main contribution of the current study was to identify and utilize the unused design space of developing gestures that are not in conflict with existing operations. We did not consider comparing Ta-Ta-tap with other stroke-based gestures. Instead, we focused on showing how well the new techniques coexist with present operations, and how well users accept those new techniques. In addition to Ta-tap and Ta-Ta-tap, we expect that the general concept of using consecutive distant taps will enable many new interaction techniques to enrich the touch screen interface.

\section{ACKNOWLEDGMENTS}

This work was supported by the IT R\&D program of MKE/KEIT. [KI10041244, SmartTV 2.0 Software Platform]

\section{REFERENCES}

1. Adams, A. and Cox, A. L. Questionnaires, in-depth interviews and focus groups. In P. Cairns and A.L. Cox, eds., Research Methods for Human Computer Interaction. Cambridge University Press (2008), 17-34.

2. Benko, H., Wilson, A., and Baudisch, P. Precise selection techniques for multi-touch screens. In proc. CHI '06, ACM (2006), 1263-1272. 
3. Bonnet, D., Appert, C., and Beaudouin-Lafon, M. Extending the Vocabulary of Touch Events with ThumbRock. In proc. GI'13, ACM (2013).

4. Boring, S., Ledo, D., Chen, X., Marquardt, N., Tang, A., and Greenberg, S. The Fat Thumb: Using the Thumb's Contact Size for Single-Handed Mobile Interaction. In proc. MobileHCI '12, ACM (2012), 39-48.

5. Davidson, P.L. and Han, J.Y. Extending 2D object arrangement with pressure-sensitive layering cues. In proc. UIST '08, ACM (2008), 87-90.

6. Findlater, L., Wobbrock, J.O. and Wigdor, D. Typing on flat glass: examining ten-finger expert typing patterns on touch surfaces. In proc. CHI'11, ACM (2011), 24532462.

7. Forlines, C., Wigdor, D., Shen, C., and Balakrishnan, R. Direct-touch vs. mouse input for tabletop displays. In proc. CHI '07, ACM (2007), 647-656.

8. Goel, M., Wobbrock, J.O., and Patel, S.N. GripSense: Using Built-In Sensors to Detect Hand Posture and Pressure on Commodity Mobile Phones. In proc. UIST '12, ACM (2012), 545-554.

9. Gustafson, S., Holz, C., and Baudisch, P. Imaginary Phone: learning imaginary interfaces by transferring spatial memory from a familiar device. In proc. UIST '11, ACM (2011), 283-292.

10. Harrison, C. and Hudson, S. Using Shear as a Supplemental Two-Dimensional Input Channel for Rich Touchscreen Interaction. In proc. CHI '12, ACM (2012), 3149-3152.

11. Harrison, C., Schwarz, J. and Hudson, S.E. TapSense: enhancing finger interaction on touch surfaces. In proc. UIST '11, ACM (2011), 627-634.

12. Heo, S. and Lee, G. Force gestures: augmenting touch screen gestures with normal and tangential forces. In proc. UIST '11, ACM (2011), 621-626.

13. Heo, S. and Lee, G. ForceTap: extending the input vocabulary of mobile touch screens by adding tap gestures. In proc. MobileHCI '11, ACM (2011), 113122.

14. Heo, S. and Lee, G. Indirect shear force estimation for multi-point shear force operations. In proc. CHI '13, ACM (2013), 281-284.

15. Hinckley, K. and Song, H. Sensor synaesthesia: touch in motion, and motion in touch. In proc. $C H I$ '11, ACM (2011), 801-810.

16. Karat, J., McDonald, J.E., and Anderson, M. A comparison of menu selection techniques: touch panel, mouse and keyboard. International Journal of ManMachine Studies 25, 1 (1986), 73-88.

17. Kin, K., Agrawala, M., and DeRose, T. Determining the benefits of direct-touch, bimanual, and multifinger input on a multitouch workstation. In proc. GI '09, Canadian Information Processing Society (2009), 119-124.

18. Microsoft MSDN, http://msdn.microsoft.com/enus/library/windows/desktop/bb760404(v=vs.85).aspx

19. Miyaki, T. and Rekimoto, J. GraspZoom: zooming and scrolling control model for single-handed mobile interaction. In proc. MobileHCI '09, ACM (2009), 11:111:4.

20. Müller, H., Gove, J., and Webb, J. Understanding Tablet Use: A Multi-Method Exploration. In proc. MobileHCI '12, ACM (2012), 1-10.

21. Myers, C.S. and Rabiner, L.R. A comparative study of several dynamic time-warping algorithms for connected word recognition. The Bell System Technical J. 60, 7 (1981), 1389-1409.

22. Research In Motion ${ }^{\mathrm{TM}}$, SurePress ${ }^{\mathrm{TM}}$ Technology. http://us.blackberry.com/specifications/communication/ surepress-touch-screen.html

23. Roth, V. and Turner, T., Bezel swipe: conflict-free scrolling and multiple selection on mobile touch screen devices. In proc. CHI '09, ACM (2009), 1523-1526.

24. Roudaut, A., Lecolinet, E., and Guiard, Y. MicroRolls: expanding touch-screen input vocabulary by distinguishing rolls vs. slides of the thumb. In proc. CHI '09, ACM (2009), 927-936.

25. Rubine, D. Specifying gestures by example. In proc. SIGGRAPH' '91, ACM (1991), 329-337.

26. Sears, A. and Shneiderman, B. High precision touchscreens: design strategies and comparisons with a mouse. International Journal of Man-Machine Studies 34, 4 (1991), 593-613.

27. Serrano, M., Lecolinet, E., and Guiard, Y. Bezel-Tap gestures: quick activation of commands from sleep mode on tablets. In proc. CHI '13, ACM (2013), 30273036.

28. Wagner, J., Huot, S., and Mackay, W. BiTouch and BiPad: designing bimanual interaction for hand-held tablets. In proc. CHI '12, ACM (2012), 2317-2326.

29. Wang, F., and Ren, X. Empirical evaluation for finger input properties in multi-touch interaction. In proc. $\mathrm{CHI}$ '09, ACM (2009), 1063-1072.

30. Wang, F., Cao, X., Ren, X., and Irani, P. Detecting and leveraging finger orientation for interaction with directtouch surfaces, In proc. UIST '09, ACM (2009), 23-32.

31. Wobbrock, J., Wilson, A., and Li, Y. Gestures without libraries, toolkits or training: a $\$ 1$ recognizer for user interface prototypes. In Proc. UIST '07, ACM (2007), 159-168.

32.Zhao, S., Maneesh, A., and Hinckley, K. Zone and polygon menus: using relative position to increase the breadth of multi-stroke marking menus, In Proc. $\mathrm{CHI}$ '06, ACM (2006), 1077-1086. 